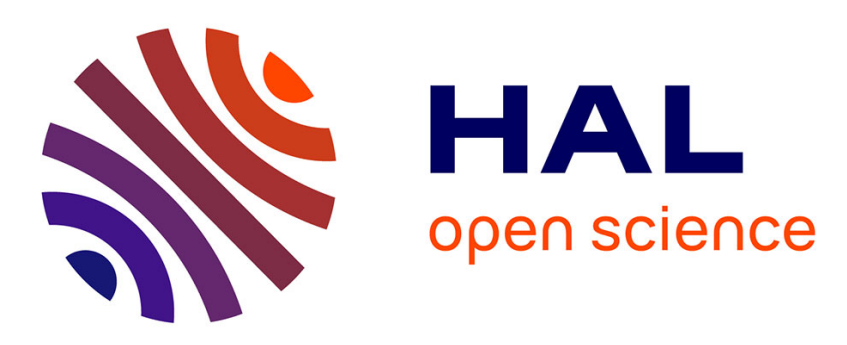

\title{
Synthesis of Optimal Electrical Stimulation Patterns for Functional Motion Restoration Applied to Spinal Cord Injured Patients
}

\author{
Mourad Benoussaad, Philippe Poignet, Mitsuhiro Hayashibe, Christine \\ Azevedo Coste, Charles Fattal, David Guiraud
}

\section{To cite this version:}

Mourad Benoussaad, Philippe Poignet, Mitsuhiro Hayashibe, Christine Azevedo Coste, Charles Fattal, et al.. Synthesis of Optimal Electrical Stimulation Patterns for Functional Motion Restoration Applied to Spinal Cord Injured Patients. Medical and Biological Engineering and Computing, 2015, 53 (3), pp.227-240. 10.1007/s11517-014-1227-x . lirmm-01235810

\section{HAL Id: lirmm-01235810 \\ https://hal-lirmm.ccsd.cnrs.fr/lirmm-01235810}

Submitted on 30 Nov 2015

HAL is a multi-disciplinary open access archive for the deposit and dissemination of scientific research documents, whether they are published or not. The documents may come from teaching and research institutions in France or abroad, or from public or private research centers.
L'archive ouverte pluridisciplinaire HAL, est destinée au dépôt et à la diffusion de documents scientifiques de niveau recherche, publiés ou non, émanant des établissements d'enseignement et de recherche français ou étrangers, des laboratoires publics ou privés. 


\title{
Synthesis of optimal electrical stimulation patterns for functional motion restoration: applied to spinal cord-injured patients
}

\author{
Mourad Benoussaad · Philippe Poignet • \\ Mitsuhiro Hayashibe • Christine Azevedo-Coste • \\ Charles Fattal · David Guiraud
}

\begin{abstract}
We investigated the synthesis of electrical stimulation patterns for functional movement restoration in human paralyzed limbs. We considered the knee joint system, co-activated by the stimulated quadriceps and hamstring muscles. This synthesis is based on optimized functional electrical stimulation (FES) patterns to minimize muscular energy consumption and movement efficiency criteria. This two-part work includes a multi-scale physiological muscle model, based on Huxley's formulation. In the simulation, three synthesis strategies were investigated and compared in terms of muscular energy consumption and co-contraction levels. In the experimental validation, the synthesized FES patterns were carried out on the quadriceps-knee joint system of four complete spinal cord injured subjects. Surface stimulation was applied to all subjects, except for one FES-implanted subject who received neural stimulation. In each experimental validation, the model was adapted to the subject through a parameter identification procedure. Simulation results were successful and showed high co-contraction levels when reference trajectories were tracked. Experimental validation results were encouraging, as the desired and measured trajectories showed good agreement, with an $8.4 \%$ rms error in a subject without substantial time-varying behavior. We updated
\end{abstract}

M. Benoussaad · P. Poignet · M. Hayashibe · C. Azevedo-Coste ·
D. Guiraud
DEMAR Group, LIRMM, INRIA, University of Montpellier 2,
CNRS, 860 Rue Saint Priest, 34095 Montpellier Cedex 5, France

M. Benoussaad $(\bowtie)$

LGP Lab. ENIT, 47 Avenue d'Azereix, 65000 Tarbes, France

e-mail: mourad.benoussaad@enit.fr

C. Fattal

PROPARA Rehabilitation Center, 263 rue du Caducée,

34090 Montpellier, France the maximal isometric force in the model to account for time-varying behavior, which improved the average rms errors from 31.4 to $13.9 \%$ for all subjects.

Keywords Rehabilitation · Functional electrical stimulation · Physiological musculoskeletal model · FES synthesis $\cdot$ Optimization techniques

\section{Introduction}

Functional electrical stimulation (FES) is generally used to activate the paralyzed muscles in spinal cord-injured subjects. This pulse-train stimulation produces muscular forces that can be controlled in a way similar to that normally occurring in the central nervous system of healthy subjects. FES can be applied directly to muscle, through the motor nerve or through the spinal cord [19]. In the first two cases, the electrodes are implanted or placed over the skin. FES can thus be used to restore functional tasks like standing or locomotion. However, the functional task needs to be clearly specified in terms of the joint trajectories to follow, the position to reach, and/or the level of torque to achieve.

In order to restore a given functional task, it is important to synthesize the best FES patterns in terms of energetic or other criteria so that the appropriate electrical stimulation patterns to restore the desired task are generated. The synthesis results can be applied in an open loop or used as part of a model-based control strategy [9]. Certainly, it should be the first step in FES-based rehabilitation for spinal cord injured subjects.

Synthesizing FES patterns is a difficult process because of the relative lack of knowledge on muscle behavior under FES, especially regarding its complexity and high nonlinearity, and very few works have focused on this issue. In 
[6, 19, 30], FES patterns were obtained empirically based only on the clinicians' experience. This intuitive solution restricts the number of activated muscles and does not ensure the best choice of patterns, which accelerate the muscle fatigue.

Functional electrical stimulation patterns were obtained in $[9,25,28]$ using an analytical inversion of a musculoskeletal model, although this model-based synthesis dealt with the activation of a single muscle only. It solved the redundancy problem by excluding any co-contraction phenomena, which is a great advantage for joint stability [24]. However, analytical inversion is usually impossible for more realistic models with high complexity and nonlinearity.

In [24], an optimization technique for FES pattern synthesis was tested in simulation. An appropriate criterion was chosen for minimization, and the method solved the redundancy problem by taking into account muscular cocontraction. However, in this work, the optimization criterion was chosen to cancel the co-contraction and exclusively solved a numerical inverse problem.

Another method for synthesis was proposed in [7] as an alternative to optimization methods. It solved the redundancy problem by partitioning the muscular actuators into two functional groupings, referred to as gravity compensators and movement generators.

This strategy required more than one muscle to perform any task, which can often be difficult to ensure in spinal cord-injured subjects. In addition, the level of co-contraction could not be explicitly controlled.

In the current work, the optimization strategy was chosen to synthesize the optimal FES patterns for movement restoration while taking into account muscular co-contraction, thus solving the redundancy problem. The main aim of this synthesis strategy is to minimize fatigue by limiting energetic consumption.

To illustrate this approach, we consider a biomechanical knee joint model and its antagonistic muscles: the quadriceps and hamstring muscle groups. In the first part of this work, several methods with different optimization criteria were explored in simulation and compared in terms of energetic and co-contraction criteria. In the second part, the synthesized FES patterns were experimentally validated on subjects with spinal cord injury. In this work, only quadriceps muscle was considered for the validation.

In the next section, the musculoskeletal model of the knee joint (Sect. 2.1), the strategies for FES pattern synthesis (Sect. 2.2) and the experimental validation (Sect. 2.3) are described. Results of the simulation study and the experimental validation are presented in Sect. 3 and discussed in Sect. 4. The conclusion and perspectives are presented in Sect. 5.

\section{Methods}

\subsection{Musculoskeletal modeling of the knee joint}

The knee joint is controlled by two antagonistic groups of electrically stimulated muscle: the quadriceps and the hamstrings. The model of this system consists of two parts [3]:

- a dynamic model of the controlled knee joint,

- a model of electrically stimulated muscle.

\subsubsection{Dynamic model of the controlled knee joint}

The knee joint is modeled in $2 \mathrm{D}$ in the sagittal plane as one degree of freedom $\theta$, as illustrated in Fig. 1a. It is controlled by quadriceps and hamstring muscular forces $F_{q}$ and $F_{h}$, through constant moment arms $r_{1}$ and $r_{2}$, respectively. The full knee extension is at $0^{\circ}$ and the rest position is at $\theta_{0}$ , which will depend on the subject. By assuming the relative foot movement with respect to the leg is very small, the shank-foot group was considered as a single rigid body. The geometrical formulations of quadriceps and hamstring muscle-tendon lengths are:

$\left\{\begin{array}{l}L_{q}(\theta)=L_{q_{\mathrm{ext}}}+r_{1} \theta \\ L_{h}(\theta)=L_{h_{\mathrm{ext}}}-r_{2} \theta\end{array}\right.$

where $L_{q_{\mathrm{ext}}}$ and $L_{h_{\mathrm{ext}}}$ are the quadriceps and hamstring lengths at the maximal extension (i.e., $\theta=0^{\circ}$ ). The knee joint dynamics around the rest position is given by the following second-order nonlinear equation $[3,27]$ :

$T_{q}-T_{h}=J \ddot{\theta}_{r}+B \dot{\theta}_{r}+K \sin \left(\theta_{r}\right)$

where $\theta_{r}=\theta_{0}-\theta$ is the knee angle from the rest position in a counterclockwise direction. $T_{q}=F_{q} r_{1}$ and $T_{h}=F_{h} r_{2}$ are the active quadriceps and hamstring torques; $B$ and $J$ are the viscosity coefficients and the shank inertia around the center of rotation $O$, respectively; $K$ is the parameter of the static torque that we considered in [3] as combining the gravity and elasticity torques.

\subsubsection{Electrically stimulated muscle model}

The muscle model highlights the multi-scale aspect of muscle as a combination of macroscopic [15] and microscopic [17] model properties by describing its dynamics from the level of fiber to the entire muscle, as detailed in a previous work [8]. The overview of this model is shown in Fig. 1b and consists of two parts:

1. The activation model, which describes the generation of action potentials and the initialization of contractions from the stimulation input. It includes two subparts: 

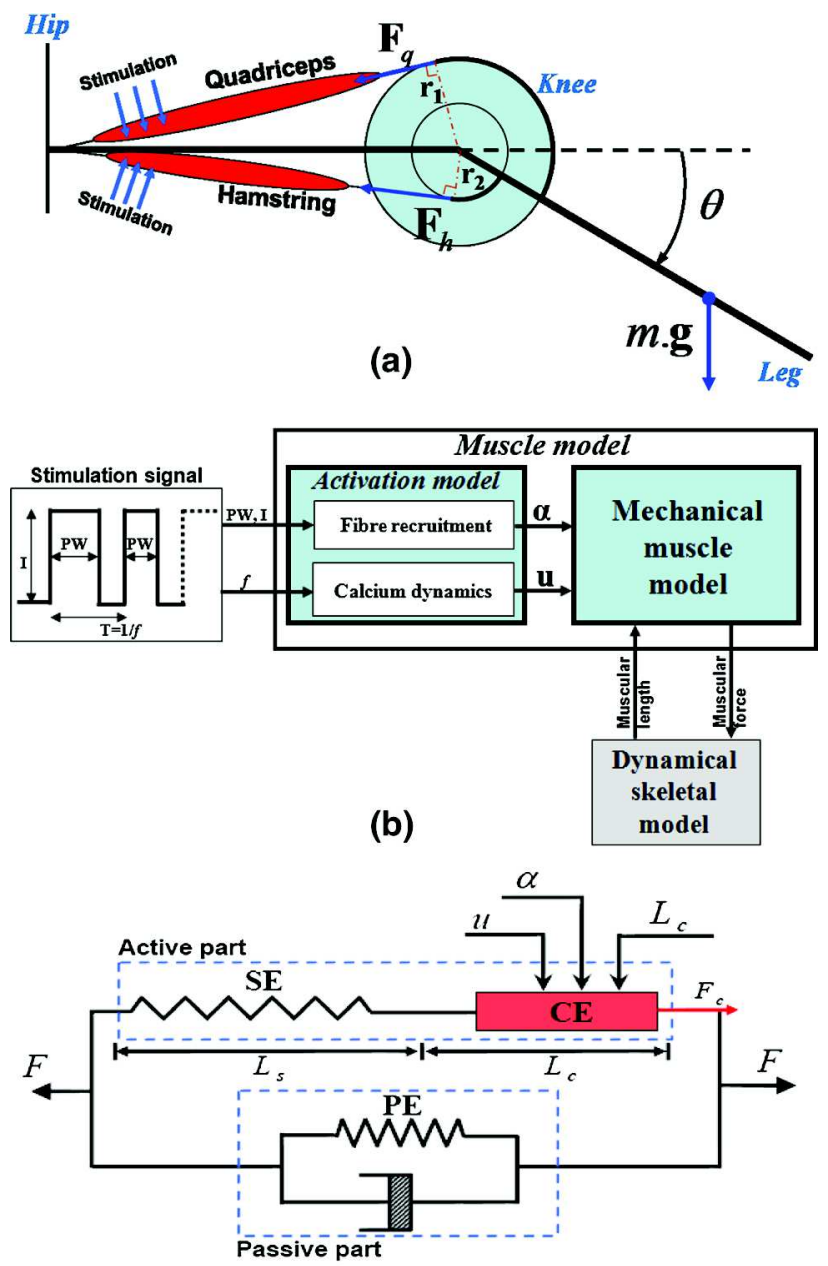

(c)

Fig. 1 Physiological model parts of the knee joint activated by two antagonistic stimulated muscles. a Biomechanical model of the knee joint, where quadriceps force $\left(\mathrm{F}_{\mathrm{q}}\right)$ and hamstring force $\left(\mathrm{F}_{\mathrm{h}}\right)$ control the knee joint angle $\theta$ through moment arms $r_{1}$ and $r_{2}$, respectively; $m . \mathbf{g}$ is the gravity torque of the leg, where $m$ is its mass and $\mathbf{g}$ the gravity acceleration; $\mathbf{b}$ overview of one muscle model. By modulating the stimulation frequency $f$, the stimulation amplitude $I$, and the pulse width $P W$, the activation model modulates the recruitment rate $\alpha$ and the chemical pulse train $u$. The stimulated muscle applies a force on the skeletal system. The joint position has feedback on the muscle length and thus on the contractile element length; $\mathbf{c}$ mechanical muscle model, which includes an elastic serial element (SE), whose stiffness is $k_{s}$ and length is $L_{s}$, a viscoelastic parallel element $(\mathrm{PE})$, and a contractile element $(\mathrm{CE})$, which generates the force $F_{c}$ and is controlled by the recruitment rate $\alpha$ and the chemical pulse train $u$ and is modulated by it length $L_{c}$

- The fiber recruitment model describes the spatial summation of activated fibers. It represents the relation between the electrical current applied to the motor point and the rate of the activated fibers $\alpha$. It is described by a sigmoid function [20] and modulated here by the electrical stimulation pulse width PW as follows:

$$
\alpha(\mathrm{PW})=\frac{\mathrm{c}_{1}}{1+\exp \left\{\mathrm{c}_{2}\left(\mathrm{c}_{3}-\mathrm{PW} / \mathrm{PW}_{\max }\right)\right\}}
$$

where $c_{1}, c_{2}$ and $c_{3}$ are the parameters that represent the plateau level, maximum slope and inflexion point, respectively.

- The calcium dynamics model represents the electrochemical phenomena responsible for the force triggering within one fiber and the temporal summation of these forces $[12,26]$. This model is controlled by the stimulation frequency and generates a chemical pulse train $u$. It is composed of three phases: muscle contraction, muscle relaxation and the transition between these two states. It is defined by the following function [8]:

$u(t)=\Pi_{c}(t) U_{c}+\left(1-\Pi_{c}(t)\right) U_{r}$

where $U_{c}, U_{r}$ are constants related to the actin-myosin cycle during the contraction, and $\Pi_{c}$ is a function defined as follows:

$\Pi_{c}= \begin{cases}1 & \text { during the contraction phase } \tau_{c} \\ \frac{\tau_{r}-t_{r}}{\tau_{r}} & \text { during the transition phase } \tau_{r} \\ 0 & \text { otherwise }\end{cases}$

where $t_{r}$ is the relative time position from the beginning of the transition phase.

2. The mechanical muscle model, which represents the contractile properties of the muscle-tendon structure. It is a Hill-type lumped element model [29] adapted for FES application. Figure 1c illustrates this model, which includes a contractile element (CE) in series with an elastic element (SE), whose stiffness is $k_{s}$, and a viscoelastic parallel element (PE). In this work, the $\mathrm{PE}$ effect is moved to the joint as a part of the passive effects, as was done in [3]. Therefore, the model of the muscle-tendon structure becomes equivalent to the active part (Fig. 1c). The specific model of CE, which describes the contraction under FES, is based on Huxley's sliding filament theory [17]. It is represented by the dynamic equations of the contractile element force $F_{c}$ and stiffness $K_{c}$, detailed in [8]:

$\left\{\begin{array}{l}\dot{K}_{c}=-\left(u+\left|\dot{\varepsilon}_{c}\right|\right) K_{c}+\alpha K_{c m} F l_{c}\left(\varepsilon_{c}\right) \Pi_{c} U_{c} \\ \dot{F}_{c}=-\left(u+\left|\dot{\varepsilon}_{c}\right|\right) F_{c}+\alpha F_{c m} F l_{c}\left(\varepsilon_{c}\right) \Pi_{c} U_{c}+K_{c} L_{c 0} \dot{\varepsilon}_{c}\end{array}\right.$

where $F_{c m}$ and $K_{c m}$ correspond to maximal isometric force and stiffness, respectively. $F l_{c}$ is the CE forcelength relationship, which relates the maximal normalized isometric force to the $\mathrm{CE}$ strain $\varepsilon_{c}$ as follows [13, 25]:

$F l_{c}\left(\varepsilon_{c}\right)=\exp \left[-\left(\frac{\varepsilon_{c}}{b}\right)^{2}\right]$

where $b$ is the so-called shape parameter, which describes the overlapping level of filaments in 
sarcomeres, and $\varepsilon_{c}=\frac{L_{c}-L_{c 0}}{L_{c 0}}$, with $L_{c}$ being the $\mathrm{CE}$ length and $L_{c 0}$ its rest length. The mechanical interaction between $\mathrm{CE}$ and $\mathrm{SE}$ allows us to formulate the $\mathrm{CE}$ deformation velocity [8]:

$\dot{\varepsilon}_{c}=\frac{k_{s} L_{0} \dot{\varepsilon}+F_{c} u-\alpha F_{c m} F l_{c}\left(\varepsilon_{c}\right) \Pi_{c} U_{c}}{k_{s} L_{c 0}+K_{c} L_{c 0}-s_{v} F_{c}}$

where:

$s_{v}=\operatorname{sign}\left(k_{s} L_{0} \dot{\varepsilon}+F_{c} u-\alpha F_{c m} F l_{c}\left(\varepsilon_{c}\right) \Pi_{c} U_{c}\right)$

and $\varepsilon=\frac{L-L_{0}}{L_{0}}$ is the strain of the whole muscle-tendon structure, with $L_{0}$ being the muscle-tendon rest length and $L=L_{c}+L_{S}$ its current length, where $L_{S}$ being the SE length.

\subsection{FES pattern synthesis}

\subsubsection{Optimization-based synthesis principle}

The optimal FES pattern synthesis consists of a modelbased optimization of the stimulation PW vectors applied to the two antagonistic muscles simultaneously. This optimization is based on the minimization of a cost function $F_{\text {cost }}$ under a set of physiological and mechanical constraints. It takes into account the entire movement duration and is stated as:

$$
\left\{\begin{array}{l}
\min _{\mathbf{P W} q, \mathbf{P W}^{i}} F_{\operatorname{cost}}\left(\mathbf{P W}^{q}, \mathbf{P W}^{i}\right) \\
s . t . \\
\mathbf{C}\left(\mathbf{P W}^{q}, \mathbf{P W}^{h}\right) \leq 0 \\
\mathbf{C}_{\mathbf{e q}}\left(\mathbf{P W}^{q}, \mathbf{P W}^{h}\right)=0
\end{array}\right.
$$

where $\mathbf{P} \mathbf{W}^{q}=\left[p w_{1}^{q}, \ldots, p w_{n}^{q}\right], \mathbf{P W}^{h}=\left[p w_{1}^{h}, \ldots, p w_{n}^{h}\right]$ are the pulse width vectors of the stimulation applied during the whole movement on the quadriceps and hamstrings, respectively. These vectors represent a discretization of continuous stimulation into $n$ samples. The choice of $n$ value was discussed in our previous work [1,2] and was fixed at $n=20$ in this work, which is enough to ensure the current desired tasks. $\mathbf{C}$ and $\mathbf{C}_{\mathbf{e q}}$ are the vectors including equality and inequality constraints such as the ranges of stimulation, joint angles, velocities and accelerations. The optimization cost function $F_{\text {cost }}$ defines the strategy of the synthesis. This choice is thoroughly discussed in the next Sect. 2.2.2.

To solve the nonlinear programming problem of optimization, the quasi-Newton algorithm was used. The stimulation vectors $\mathbf{P} \mathbf{W}^{q}, \mathbf{P} \mathbf{W}^{h}$ were initialized to $10 \%$ of their maximal value $P W_{\max }$. The simulation and optimization algorithms were performed using MAtLAB 7.0.0 on a PC platform (Pentium-IV 3-GHz, 1-GB RAM). The numerical integration of the model dynamics was performed through the MATLAB solver "ODE113" with a variable step-size.
The optimal FES patterns were synthesized off-line and then applied, as stimulation input, to the model for simulation (Sect. 3.1) and then to a real subject for experimental validation (Sect. 3.2).

\subsubsection{Optimization-based synthesis strategies}

In this simulation study, we investigated different cost function choices and their effects on movement efficiency, energy consumption and the co-contraction phenomenon, as in our previous work [2]. For this study, the hamstring muscle parameters were taken from the literature, whereas the quadriceps and knee joint parameters were identified experimentally [3], as presented below (Sect. 2.3.3). In addition, the rest position of the leg was to be assumed vertical, i.e., $\theta_{0}=90^{\circ}$. The following three synthesis strategies were thus applied:

- The first synthesis strategy generated FES patterns that allowed tracking a fifth-order polynomial trajectory by the knee joint. This trajectory is generally usually used in robotics to perform a movement from an initial to a final joint state [18]. Therefore, the quadratic cost function includes one part related to trajectory tracking errors and another part related to muscular activations. It was assessed throughout the movement duration from 0 to $t_{\text {end }}$ :

$$
\begin{aligned}
F_{\text {cost }}= & \int_{t=0}^{t_{\text {end }}} w_{\text {err }}\left(\theta_{s}(t)-\theta_{\text {ref }}(t)\right)^{2} d t \\
& +\int_{t=0}^{t_{\text {end }}} w_{q} \alpha_{q}^{2}(t)+w_{h} \alpha_{h}^{2}(t) d t
\end{aligned}
$$

where $\theta_{\text {ref }}$ and $\theta_{s}$ represent the polynomial reference trajectory and the simulated knee joint trajectory. $\alpha_{q}$ and $\alpha_{h}$ are the recruitment fiber rates in the quadriceps and the hamstring muscles, which correspond to each muscular activation. $w_{q}, w_{h}$ and $w_{\text {err }}$ are the weight coefficients that affect the tracking accuracy and the co-contraction rate, as will be discussed further on (Sect. 4).

- In the second synthesis strategy, instead of using a predefined polynomial joint reference trajectory, it was optimized based on the energetic criterion of knee joint torque and defined as:

$F_{\text {traj }}=\int_{t=0}^{t_{\text {end }}}\left|\Gamma_{a}(t) \times \dot{\theta}_{\text {ref }}(t)\right| d t$

where $\Gamma_{a}$ is the resultant of the antagonistic muscular torques. Thereafter, the FES patterns were synthesized using the same optimization as in the first strategy (Eq. 11 ) by considering the optimal trajectory as reference one $\left(\theta_{\text {ref }}\right)$. 
- In the third strategy, we avoided using a reference trajectory as for several functional tasks, only certain joint situations need to be reached. Therefore, this synthesis generated FES patterns to perform a movement from an initial to a final joint state. In this case, the cost function included muscular activities only:

$$
F_{\text {cost }}=\int_{t=0}^{t_{\text {end }}}\left(w_{q} \alpha_{q}^{2}(t)+w_{h} \alpha_{h}^{2}(t)\right) \mathrm{dt}
$$

As no reference trajectory had to be tracked, we defined a set of constraints in order to reach the desired final situation and with respect to the angle and velocity boundaries. These constraints can be summarized below:

$$
\left\{\begin{array}{l}
{\left[\theta_{s}^{f}, \dot{\theta}_{s}^{f}\right]=\left[\theta_{d}^{f}, \dot{\theta}_{d}^{f}\right]} \\
\theta_{\min }<\theta_{s}<\theta_{\max } \\
\left|\dot{\theta}_{s}\right| \leq \dot{\theta}_{\max } \\
\operatorname{sign}\left(\dot{\theta}_{s}\right)=\operatorname{sign}\left(\theta_{d}^{f}-\theta^{i}\right)
\end{array}\right.
$$

where $\theta_{s}^{f}, \theta_{d}^{f}$ are the simulated and desired final angles and $\dot{\theta}_{s}^{f}, \dot{\theta}_{d}^{f}$ are the simulated and desired final velocities. $\left[\theta_{\min }, \theta_{\max }\right]$ are the angular boundaries, $\dot{\theta}_{\max }$ is the maximal velocity, and $\theta^{i}$ is the initial angular position. The last constraint was added to define only one direction of movement and avoid any oscillation, where sign represents the sign function.

\subsubsection{Energetic criteria and co-contraction rate}

To compare these three strategies, we defined two energetic criteria and one co-contraction level criterion, which were evaluated throughout the entire duration of movement. For the first energetic criterion $E_{\text {art }}$, the resultant of the antagonistic muscular torques around the knee joint was considered:

$E_{\text {art }}=\frac{1}{t_{\text {end }}} \int_{t=0}^{t_{\text {end }}}\left|\left(F_{h}(t) \times r_{2}-F_{q}(t) \times r_{1}\right) \times \dot{\theta}(t)\right| \mathrm{dt}$

The second energetic criterion $E_{\text {mus }}$ included the quadratic sum of the antagonistic muscle contributions:

$E_{\mathrm{mus}}=\frac{1}{t_{\mathrm{end}}} \int_{t=0}^{t_{\mathrm{end}}} \alpha_{q}(t)^{2}+\alpha_{h}(t)^{2} \mathrm{dt}$

To perform a more complete comparison, we had to evaluate the co-contraction rate $R_{\text {co }}$ for each strategy. Therefore, we defined a criterion based on the comparison between the antagonistic muscular torque $\left(T_{h}, T_{q}\right)$ contributions:

$R_{\mathrm{co}}(t)=1-\frac{\left|T_{h}-T_{q}\right|}{T_{h}+T_{q}}$

For this criterion, close torques indicated high co-contraction rates, while a distant torque indicated a low co-contraction rate. This criterion was normalized for the amount of torque, such that 1 corresponded to the maximal co-contraction rate and 0 corresponded to the minimal rate.

\subsection{Experimental validation of FES synthesis}

\subsubsection{Reduced musculoskeletal system}

The main problems in experimentally validating the FES synthesis were related to the parameter estimations of the real musculoskeletal system. These estimations were made in our previous work [3] on the quadriceps muscle. Therefore, only the quadriceps muscle was stimulated for the knee extension in the current work, while the hamstring muscle was kept passive and the knee flexion was performed by leg gravity.

The synthesis for this experimental validation was similar to the first strategy (Sect. 2.2.2). However, we defined a different, repetitive and more complex reference trajectory as an application of the tracking trajectory task. Since the optimization was applied to this reduced model (quadriceps-shank system), the optimization cost function ignored the hamstring activities $\alpha_{h}$ in Eq. 11 and thus became:

$$
F_{\text {cost }}=\int_{t=0}^{t_{\text {end }}} w_{\text {err }}\left(\theta_{s}(t)-\theta_{\text {ref }}(t)\right)^{2} \mathrm{dt}+\int_{\mathrm{t}=0}^{\mathrm{t}_{\text {end }}} \mathrm{w}_{\mathrm{q}} \alpha_{\mathrm{q}}^{2}(\mathrm{t})
$$

\subsubsection{Experimental setup}

Experiments were conducted at the PROPARA Rehabilitation Center (Montpellier, France) on four male subjects having complete spinal cord lesion, with an ASIA A score. We obtained approval from the local ethics committee and informed, signed consent from the patients before the experiments. Details of the patients' clinical assessments are summarized in Table 1.

The subjects were seated in a chair with the hips flexed at approximately $90^{\circ}$ and the thighs and back held against the seat. Therefore, since the hip flexion was fixed, the rectus femoris, which is a biarticular muscle, acted as a monoarticular muscle of the knee joint within the quadriceps group. The right and left quadriceps-shank units were considered separately, and the test order of quadriceps-shank side (right or left) was randomized.

The quadriceps muscle group was stimulated with the PROSTIM stimulator (Hardtech, Montpellier, France) through surface electrodes. However, neural stimulation was performed using an implanted FES system for subject 10 [11]. PROSTIM stimulator provides 8 stimulation channels, for which the frequency $f$, the amplitude $I$ and the pulse with $P W$ can be independently controlled. It offers the possibility to vary in real time the stimulation parameters during the same patterns of stimulation, which is not 
Table 1 Subjects' characteristics

\begin{tabular}{lllllll}
\hline Number $^{\mathrm{a}}$ & Subject $^{\mathrm{b}}$ & Age (years) & Weight $(\mathrm{kg})$ & Height $(\mathrm{m})$ & Injury level & Post-injury (years) \\
\hline 6 & FC & 34 & 50 & 1.69 & T6 & 5 \\
7 & AV & 23 & 54 & 1.72 & C7 & 2.5 \\
9 & PC & 22 & 62.7 & 1.91 & C5 & 1 \\
$10^{\text {c }}$ & MM & 48 & 75 & 1.75 & T6 & 19 \\
\hline
\end{tabular}

a Number of subjects according to numbering used in [3]

b Subject appellation, corresponds to initial of name

c Subject with FES-implanted system [11]

Fig. 2 Experimental setup for dynamical measurements: a Sensor and data acquisition system for joint angle measurements. b Muscle stimulation devices

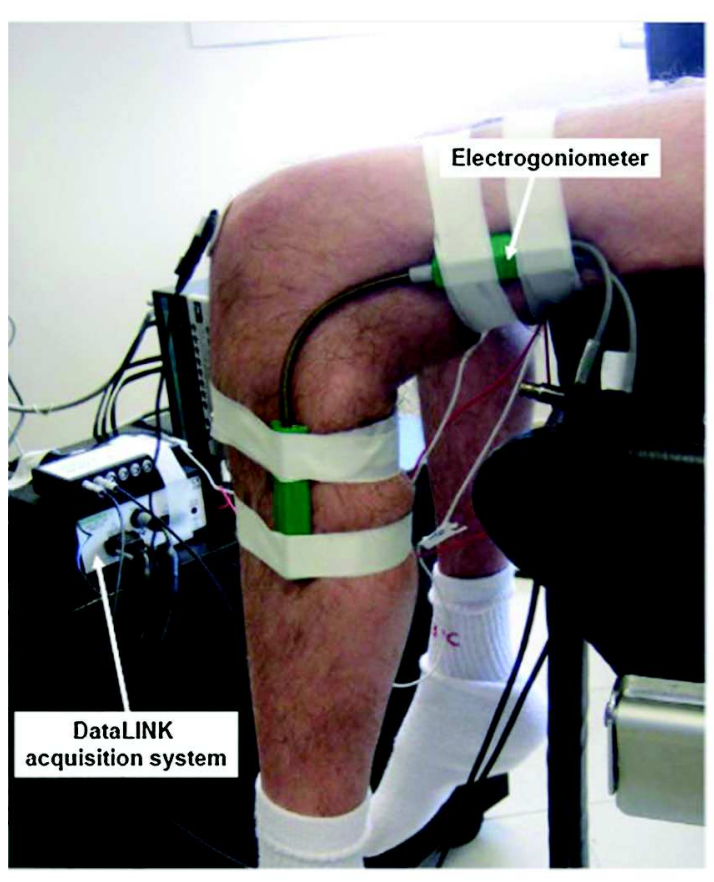

(a)

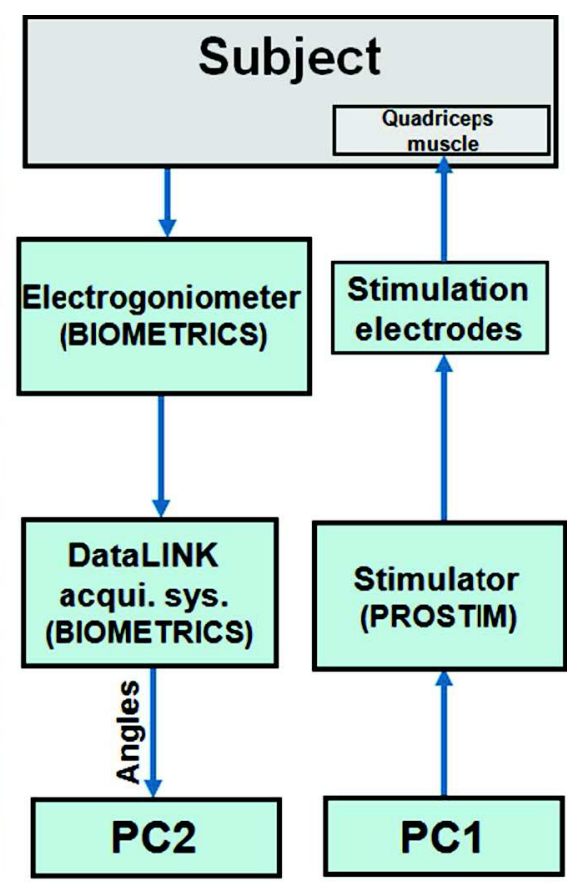

(b) the case of most of existing stimulator. One of the two surface electrodes for stimulation $(10 \mathrm{~cm} \times 5 \mathrm{~cm}$, Cefar Medical, Lund, Sweden) was placed at the top of the rectus femoris and the other was placed at the bottom of the vastus medialis, in order to stimulate the entire quadriceps muscle group.

The stimulation frequency was set at $20 \mathrm{~Hz}$ for all subjects. This was chosen to induce smooth contractions with the lowest fatigue effect possible according to [4]. The stimulation amplitude (current intensity) was obtained individually for each subject during a pre-experiment by setting the pulse width to $300 \mu$ s and increasing the amplitude until level 3 of the Medical Research Council scale was reached. The quadriceps force was controlled by modulating the pulse width, which was limited to $\mathrm{PW}_{\max }=420 \mu \mathrm{s}$ for the patients safety.

This experimental validation required two successive parts. The first concerned the estimations of model parameters in both isometric and dynamic conditions, as in our previous work [3], and is summarized in the next Sect. 2.3.3. In this first part, the torque was measured in isometric conditions using the BIODEX dynamometer (Biodex 3, Shirley Corporation, NY, USA) and recorded on a PC through the Biopac MP100 acquisition device (Biopac Systems, CA, USA) and the isolation units INISO-Biopac. This experimental procedure is thoroughly detailed in [3].

In the second part, the model identified for a specific subject was used to optimize the FES patterns according to the Eq.18 criterion. These synthesized FES patterns were then applied to the quadriceps muscle of the same subject through the PROSTIM stimulator (see Fig. 2b) and to its identified model to obtain the simulation. This experimental validation, which was the main purpose of this part of our work, was conducted in dynamic leg conditions. Thus, the knee joint angles were acquired using an externally mounted electrogoniometer (BIOMETRICS Ltd, 
VA, USA), as presented in Fig. 2a, and recorded on a PC through the DataLINK acquisition system (Fig. 2b).

Thereafter, the knee joint angles and joint torques were sampled at $100 \mathrm{~Hz}$ and $2 \mathrm{kHz}$, respectively and then lowpass filtered at $30 \mathrm{~Hz}$ using the fourth-order Butterworth filter, as in [10,27].

\subsubsection{Parameter identification procedure}

Parameter estimation, which adjusts the model parameters that account for subject specificities, was required to obtain the most realistic subject-specific behavior. As mentioned before, this parameters estimation procedure was detailed in our previous work [3] and applied to the reduced musculoskeletal system (Sect. 2.3.1). It includes five successive steps, summarized below:

(a) The geometrical parameters $L_{q_{\mathrm{ext}}}$ and $r_{1}$ of Eq. 1 were estimated without any limb measurement. Indeed, for several joint angles, their corresponding quadriceps lengths were obtained using Hawkins' equation [14] and subject height.

(b) The knee joint mechanical parameters (Eq. 2), which are the inertia $J$, the viscosity coefficient $B$, and the coefficient of the static torque $K$, were estimated without any quadriceps activation (i.e., $\mathrm{T}_{\mathrm{q}}=0$ ) through two successive steps. In the first one, the static leg torques (i.e., $\dot{\theta}_{r}=0$ ) were measured for different knee joint angles, from the rest position to full extension with steps of $5^{\circ}$, using the BIODEX system. From these measurements and Eq. 2, $K$ was estimated using a linear least square method. In the second step, the dynamic leg behavior was acquired during the passive pendulum test. As the measured kinematic data of the leg oscillations presented second-order system behavior, an analytical analysis of the damping ratio and natural frequency enabled parameter estimation of $J$ and $B$ [21].

(c) To estimate the parameters of the force-length relationship (Eq. 7), the isometric torques at different knee joint angles were measured at the same quadriceps stimulation level. Thus, these Gaussian function parameters were identified using a linear least square method.

(d) The recruitment function parameters (Eq. 3) were estimated from the measured isometric torques for different quadriceps stimulation levels at the same optimal knee joint position, which corresponds to the maximal force during the force-length estimation.

(e) The mechanical parameters of muscle were estimated in dynamical leg conditions, while the quadriceps was stimulated with the chosen stimulation-level profile. From the stimulation input and the kinematic measure- ments of the leg, estimation based on the resolution of the nonlinear programming problem was applied [3].

\section{Results}

\subsection{Simulation results for the complete system}

To appropriately compare the three synthesis strategies (Sect. 2.2.2), the same knee joint conditions (initial and final angles and velocities) were chosen. In each of these simulations, the knee joint starts at the rest position (i.e., $90^{\circ}$ ) and reaches $30^{\circ}$ without any initial or final velocities. Unless otherwise specified, the weight coefficients (Eq. 11) were set as $w_{q}=w_{h}=w_{\text {err }}=1$, called here weightingl.

The results of the first synthesis, based on polynomial trajectory tracking, are presented in Fig. 3, left column. The knee joint angle trajectories (Fig. 3g) show good agreement between the simulated and reference polynomial trajectories. This result required only 20 pulse width samples, which were normalized as shown in Fig. 3a. The antagonistic muscular torques (Fig. 3d) were close during the first, second and then the quadriceps torque became bigger. However, the two torques had the same dynamics during the entire movement.

Figure $3 \mathrm{a}-\mathrm{d}$ also indicate the appearance of the cocontraction phenomenon. We believe that its high level is related to trajectory tracking errors in the cost function (Eq. 11) and thus to the choice of weight coefficients. Therefore, we investigated the weight coefficient effects on the co-contraction rate and tracking accuracy. Subsequently, this first synthesis strategy was also tested with another weight coefficient set, called weighting2 and defined as $w_{\text {err }}=1, w_{q}=w_{i}=10$. The results with weightingl and weighting 2 were then compared. Figure $4 \mathrm{a}$ shows the co-contraction rates (Eq. 17) for each of these weight coefficient sets and highlights the lower co-contraction rate with weighting2, although its tracking errors are clearly bigger, as presented in Fig. 4 b.

The second synthesis results are summarized in Fig. 3, middle column. Similar to the first method, there was good agreement (Fig. 3h) between the optimized trajectory (Eq. 12) and the simulated one obtained by applying the synthesized stimulation pulse widths (Fig. 3b). The antagonistic muscular torques, presented in Fig. 3e, show the same dynamics for the two torques and the close levels during the first $0.5 \mathrm{~s}$.

The third synthesis results are summarized on Fig. 3, right column, where the final knee joint conditions (i.e., $\theta=30^{\circ}$ and $\dot{\theta}=0$ ) were reached without tracking any explicit trajectory (Fig. 3i). The synthesized stimulation pulse widths (Fig. 3c) and the antagonistic muscular torques 


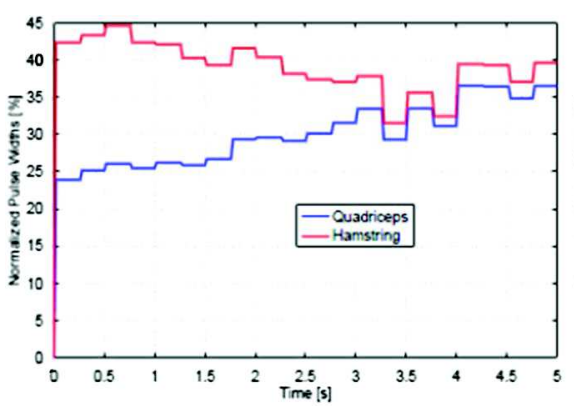

(a)

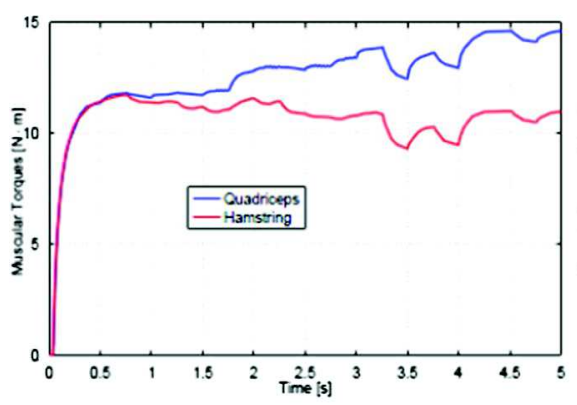

(d)

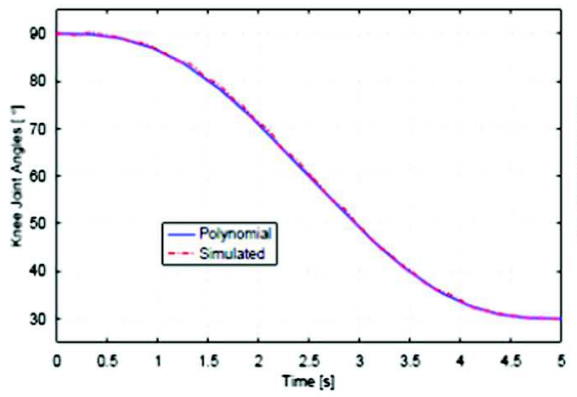

(g)

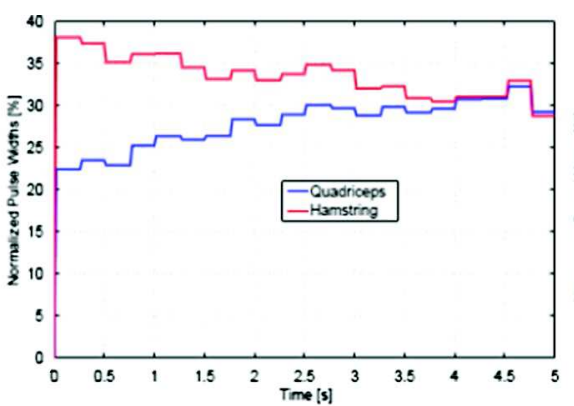

(b)

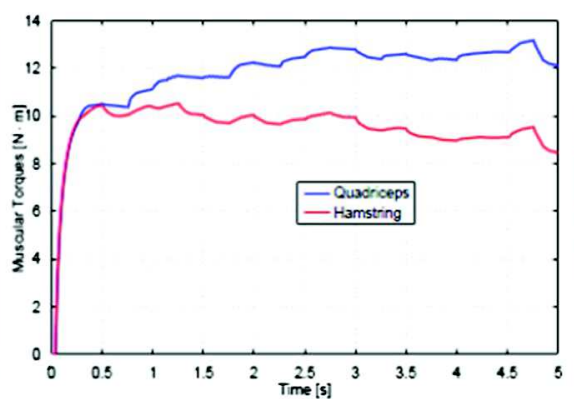

(e)

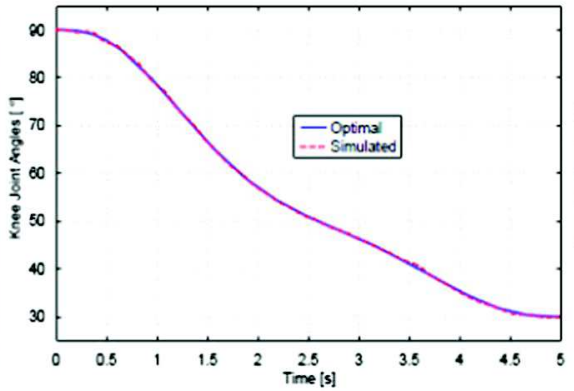

(f)

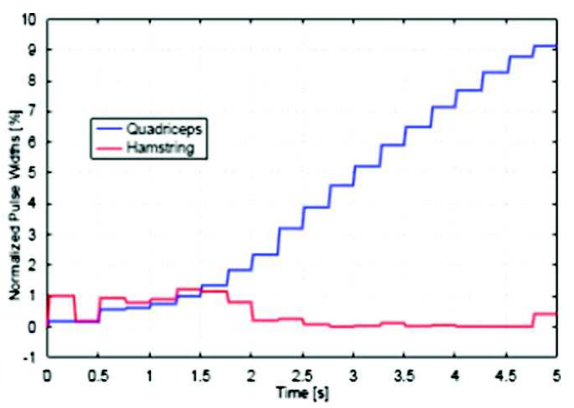

(c)

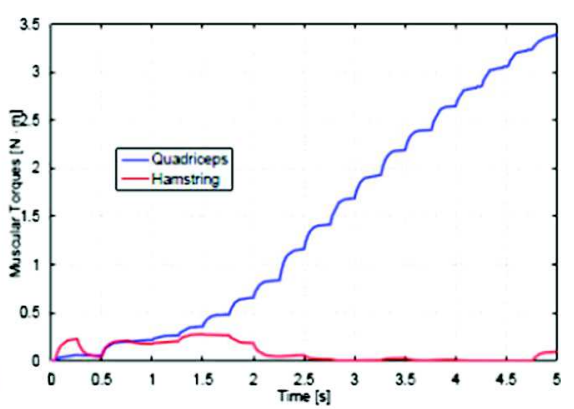

(f)

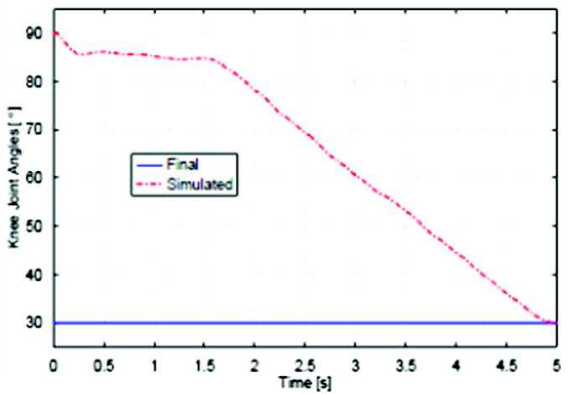

(g)

Fig. 3 Results for the three synthesis methods by: tracking a polynomial reference trajectory (a, d, $\mathbf{g}$ ); tracking an optimized reference trajectory $(\mathbf{b}, \mathbf{e}, \mathbf{h})$ and without tracking any reference trajectory $(\mathbf{c}, \mathbf{f}, \mathbf{i})$

(Fig. 3f) indicate that the contribution of the quadriceps was greater than that of the hamstrings, corresponding to the direction of movement. This difference highlights a small muscular co-contraction, which will be discussed further on.

The three criteria, defined by Eqs. 15-17, were evaluated for each synthesis method. The co-contraction rate curves are presented in Fig. 5a, while the joint energetic criterion $E_{\text {art }}$ and the muscular energetic criterion $E_{\text {mus }}$ are quantified, normalized and illustrated on the left and right sides of Fig. 5b, respectively.

\subsection{Experimental validation results with the reduced system}

In our previous work on parameter identification [3], the validation results indicated the good identification performance for most of the subjects. Each successfully identified model was then used in the synthesis procedure (Sect. 2.3) for the subject under consideration, and then, the leg that presented acceptable experimental responses was chosen for the validation. Therefore, only one leg was considered for each subject except for subject 9, whose two legs were tested. In order to evaluate the synthesis result performance, we defined an error criterion, called the normalized root mean square deviation (NRMSD), which quantified the deviation between the reference and measured knee joint trajectories, as follows:

$$
\operatorname{NRMSD}=\frac{\sqrt{\frac{1}{N} \sum_{i=1}^{N}\left(X_{m}-X_{\text {ref }}\right)^{2}}}{\left|\max \left(X_{\text {ref }}\right)-\min \left(X_{\text {ref }}\right)\right|} \times 100 \%
$$



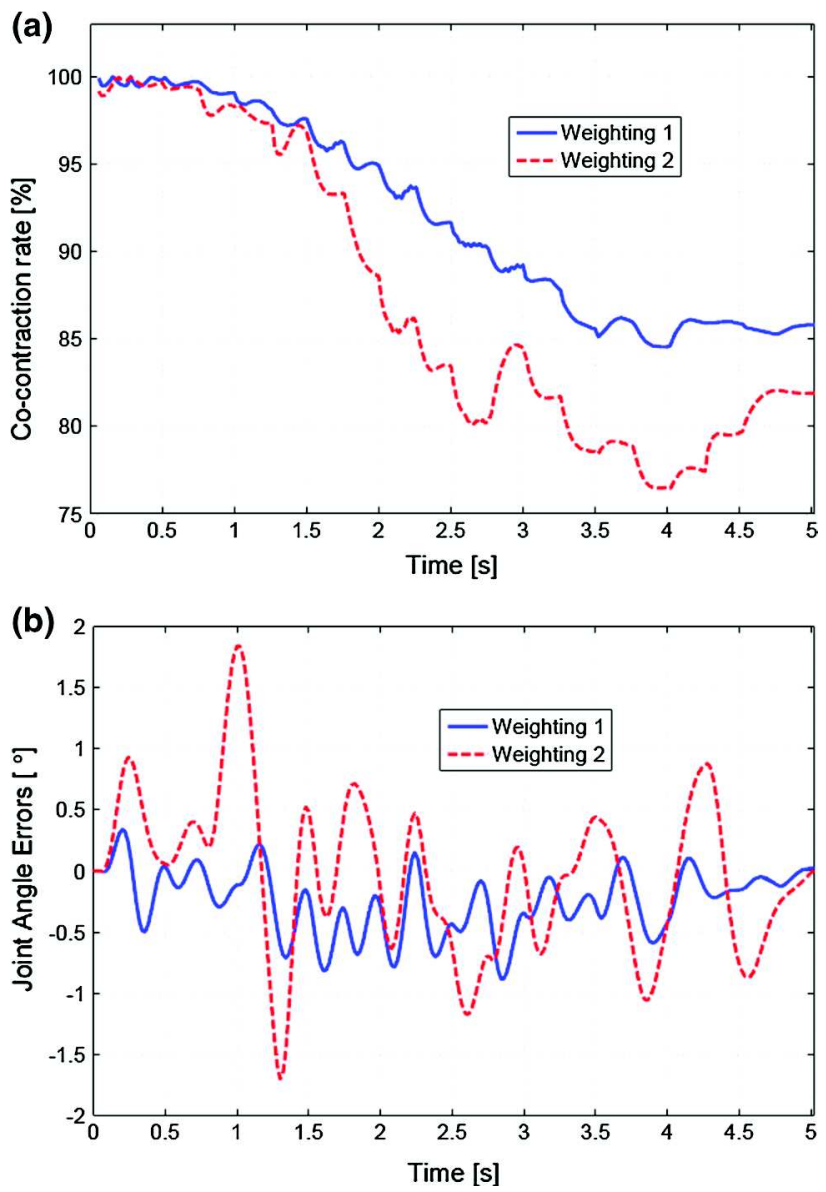

Fig. 4 Weight coefficient effects on a the co-contraction rate and $\mathbf{b}$ the joint angle tracking errors

where $X_{m}$ and $X_{\text {ref }}$ are the vectors of the measured and reference knee joint trajectories, respectively, and $N$ the number of samples within the considered duration.

The results analysis during the identification and synthesis validation experiments indicated two behavior models, depending on the quadriceps-shank system.

\subsubsection{Time-invariant model}

Initially, the system behavior was considered as having a neglected time-variation, and thus, all phenomena like fatigue, potentiation and other variations related to electrode placement were neglected. Therefore, the model's identified parameters were considered to be constants, as adjusted during the identification protocol [3].

Figure 6, left column, summarizes the FES synthesis validation results using the time-invariant model (i.e., with a time-invariant identified parameter) on the left quadriceps-shank of subject 6 . The synthesized stimulation, whose pulse widths are illustrated in Fig. 6a, was applied

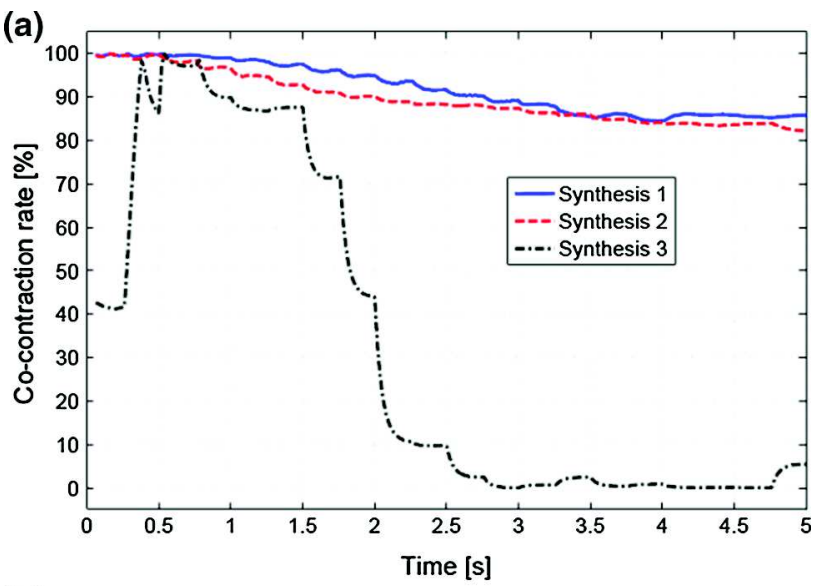

(b)

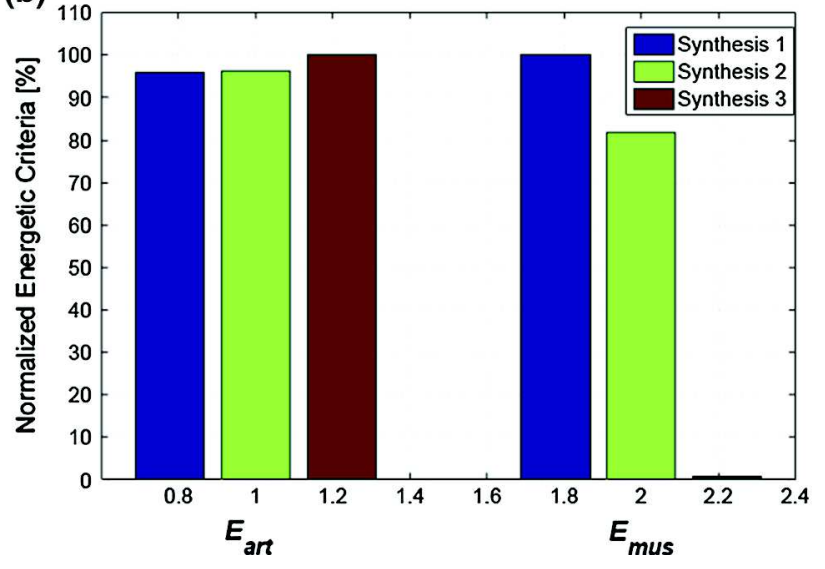

Fig. 5 Comparison of the three synthesis strategies in terms of a cocontraction rate and $\mathbf{b}$ energetic criteria

to the left quadriceps of subject 6 and, as an input, to its model to simulate the knee joint trajectory. Figure $6 \mathrm{c}$ illustrates the reference trajectory used for this synthesis, the measured knee joint trajectory and the simulated trajectory. Figure 6e shows angular errors between the measured and the reference knee joint trajectories, with an NRMSD (Eq. 19) of around $8.4 \%$.

\subsubsection{Time-variant model}

For the other experimental cases, time-varying behavior was observed between the identification session and the synthesis validation session, an example being the right quadriceps-shank of subject 9, whose synthesis validation results are presented in Fig. 6, right column. In order to analyze and quantify this variation, the same stimulation profile used to identify the quadriceps mechanical parameter (Sect. 2.3.3e) was applied again at the beginning of the synthesis validation experiments. Indeed, in the case of the right quadriceps-shank of subject 9 , a variation in behavior 
(a)

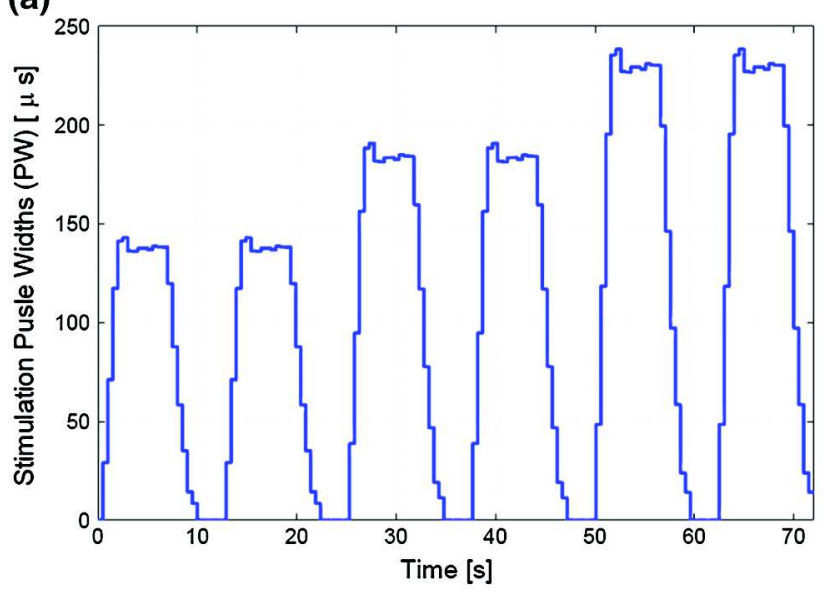

(c)

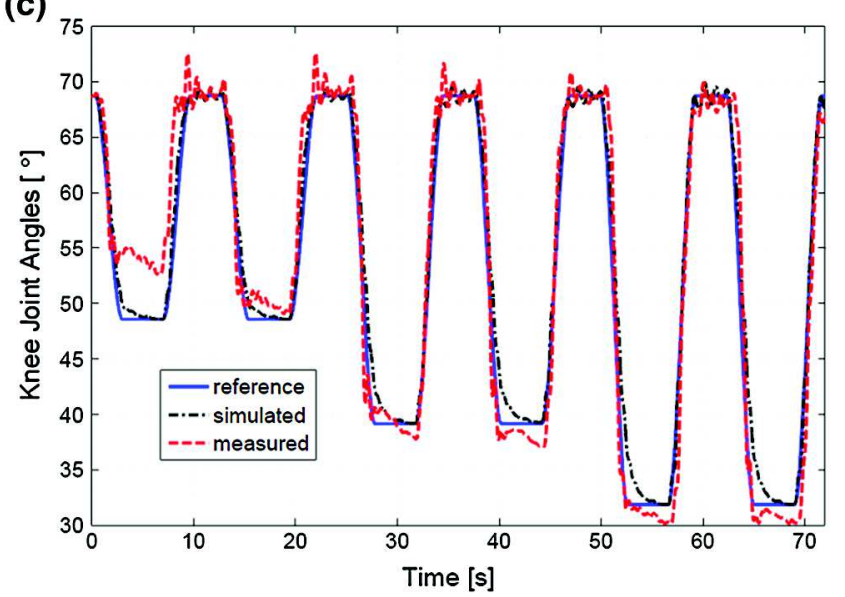

(e)

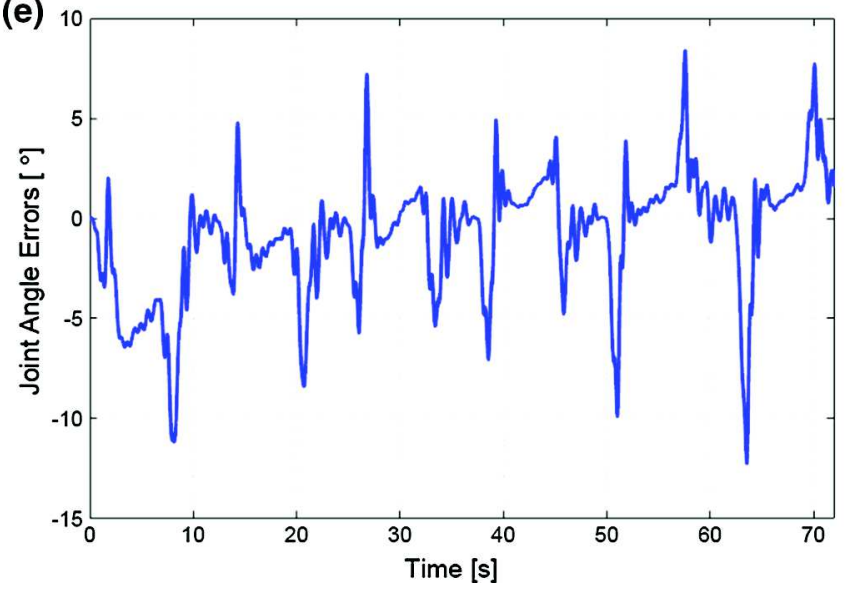

Fig. 6 FES synthesis experimental validation: with a time-invariant model (subject 6-left leg) (a, c, e) and with a time-variant model (subject 9-right leg) (b, d, f). Synthesized stimulation pulse widths (a, b); knee joint angle trajectories (reference, simulated and measured)

was observed under the same stimulation profile, indicating that the time-invariant model was inadequate to account for this system behavior. However, since the stimulation (b)

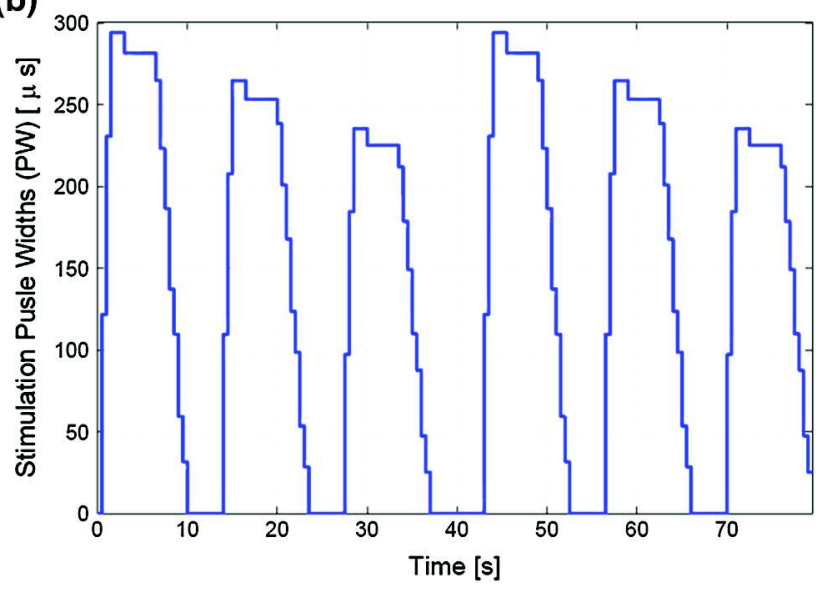

(d)

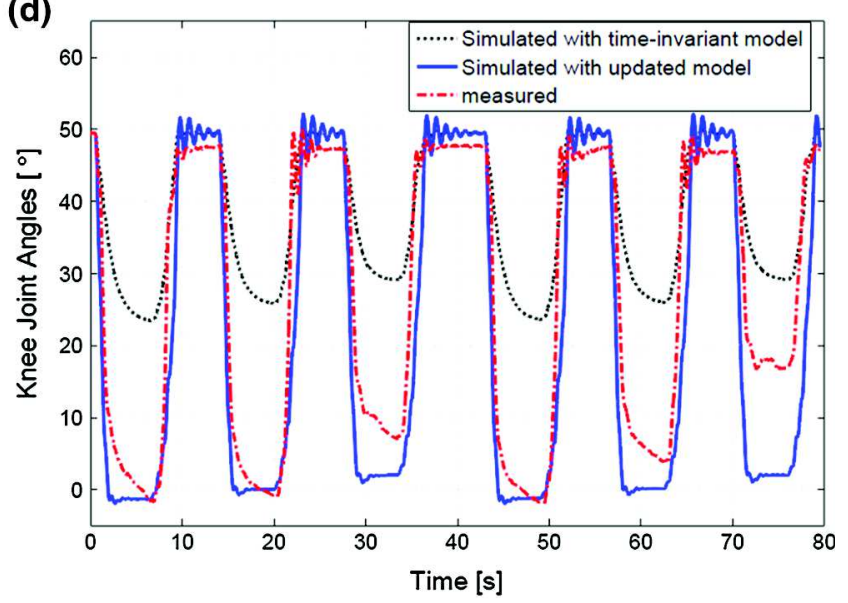

(f)

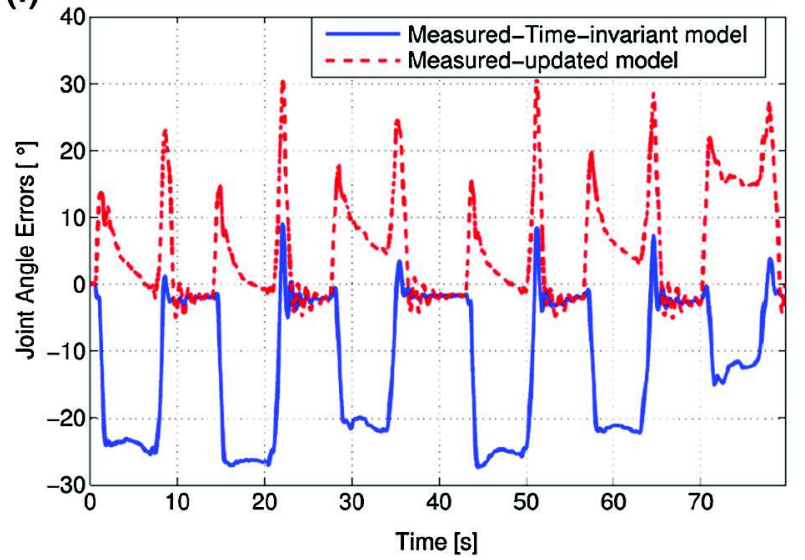

(c) or (simulated with two models and measured) (d); angular errors between measured and reference trajectories (e) or between measured and simulated trajectories (f)

patterns were synthesized off-line before the synthesis validation session, this model was still used for the FES synthesis. 
Table 2 Maximal isometric force $F_{\mathrm{cm}}$ (originally identified and updated) and its corresponding NRMSD of errors between desired and measured trajectories of synthesis results in all considered subjects

\begin{tabular}{llllllll}
\hline Model version & Synthesis errors and the updated parameter & $\mathrm{S}^{\mathrm{a}}$ & $\mathrm{S} 7 \mathrm{~L}$ & $\mathrm{~S}^{\mathrm{a}} \mathrm{R}^{\mathrm{b}}$ & $\mathrm{S} 9 \mathrm{~L}$ & S10R & NRMSD average \\
\hline Firstly identified & NRMSD $(\%)$ & 8.46 & 42.9 & 55.9 & 13.5 & 36.6 & 31.42 \\
model $^{\mathrm{c}}$ & $\mathrm{F}_{\mathrm{cm}}(\mathrm{N})$ & 270 & 269 & 126 & 500 & 300 & \\
Updated model & NRMSD $(\%)$ & 8.46 & 21.6 & 13.2 & 9.38 & 17.2 & 13.96 \\
& $\mathrm{~F}_{\mathrm{cm}}(\mathrm{N})$ & $270^{\mathrm{d}}$ & 100 & 400 & 420 & 100 & \\
\hline
\end{tabular}

a S6L: Left quadriceps-shank of subject 6

b S7R: Right quadriceps-shank of subject 7

${ }^{c}$ Model obtained after the identification procedure, as detailed in [3]

d The update did not change the $F_{c m}$ value

The application of these synthesized FES patterns (see Fig. 6b) to the real quadriceps and the time-invariant model allowed us to obtain, respectively, the measured knee joint trajectory (dashed red line) and the simulated knee joint trajectory (with the time-invariant model; dotted black line), as shown in Fig 6d, which confirmed the time-variation behavior observed before. This variation appeared to be a difference in movement amplitude with, however, the same dynamics. From this observation, we assumed that the variation was mainly due to the quadriceps maximal isometric force $\left(F_{\mathrm{cm}}\right)$ variation. Therefore, we updated this parameter such that the two responses, obtained during the two experimental sessions under the same stimulation profile, became as close as possible. This updated model was used to simulate the updated knee joint trajectory (solid blue line), which showed better agreement with the measured trajectory (Fig. 6d). However, we noted a bigger difference at the last motion pattern, as presented by the errors between these updated simulated and measured trajectories (Fig. 6f). Taking into account these two behaviors, the synthesis result errors (NRMSD) between the desired trajectories and those actually measured in all subjects are summarized in Table 2. For the quadriceps-shank systems that presented time-varying behavior and thus required a model update, the errors and maximal isometric force with the first-identified model and with the updated one (i.e., updated $F_{\mathrm{cm}}$ value) are presented in the same column (see Table 2).

\section{Discussion}

In the first synthesis strategy, the antagonistic muscular torques (Fig. 3d) highlighted the expected high quadriceps torque, which corresponded to the direction of movement. However, during the first second of movement, the two antagonistic torques were close, while their stimulation pulse widths were different. This is due to the characteristic differences between the quadriceps and the hamstring muscles. Besides, these torques had the same dynamics with different levels, since the resultant torque was smooth and presented no abrupt change. The number of control samples $n$ depends on the entire movement duration and the complexity of the trajectory to track. In this first simulation, the choice of 20 samples led to $0.25 \mathrm{~s}$ per sample, which was satisfactory. Therefore, increasing the movement duration or the complexity of the trajectory may require increasing the sample number to maintain the same accuracy; however, this would increase the calculation time. The results for this first strategy highlight the effectiveness of the optimization method and its feasibility for synthesizing stimulation patterns. The choice of this reference trajectory can nevertheless be inappropriate for movement performance from the desired initial joint situation to the final joint situation, since it introduces an excessive co-contraction ratio. However, this strategy may be used for special applications like obstacle avoidance or grasping tasks to accurately follow a desired trajectory.

The results on the weight set comparisons (Fig. 4) show that the FES synthesis with weighting2 minimized the cocontraction rate but increased the tracking errors. This was due to the importance in the cost function given to muscular activities compared with errors. Therefore, the use of this method requires a compromise between the desired tracking accuracy and the co-contraction level, which is directly related to the muscular energetic consumption, as discussed further on.

The results for the second synthesis present several similarities with those of the first synthesis, in terms of co-contraction level. Indeed, even though this strategy first minimized the energy at the torque level, it imposed a tracking accuracy criterion in the second step of the optimization, thereby making its co-contraction results similar to the first strategy results. This finding indicates that trajectory tracking is the reason for the co-contraction level, independently of the trajectory type. On the other hand, the normalized pulse widths (Fig. 3c) and antagonistic muscular torques (Fig. 3f) of the third synthesis method illustrate 
a short period and low co-contraction level. Indeed, the co-contraction rates (Fig. 5a) illustrate a higher muscular co-contraction level in the two first synthesis methods than that in the third one. This resulted in a large gap in muscular activity consumption between the first two methods and the third one, as shown by their muscular activation criterion $E_{\text {mus }}$ (right side of Fig. 5b), although their values for the joint-level energetic criterion $E_{\text {art }}$ are quite close (left side of Fig. 5b). This also showed the direct proportion between co-contraction levels and the muscular consumption criterion.

These comparisons revealed that the synthesized stimulation patterns of the first and second methods are not optimal in terms of muscular activity consumption. Therefore, the FES synthesis based on trajectory tracking, which is generally used in robotics, is unsuitable for a musculoskeletal antagonistic system, except when trajectory tracking is absolutely required. In the case of trajectory tracking, this occurs under natural control conditions and the universal experience is that high tracking precision requires highenergy consumption and therefore induces greater muscular fatigue.

The low co-contraction level in the third method had the advantage of low muscular consumption, due to the low cocontraction rate. However, the co-contraction phenomenon, often observed in natural systems, can be very useful for increasing active joint stiffness. This stiffness maintains joint stability [24] in situation of external perturbation, such as ground contact during walking. In future works, a compromise must be found between low muscular energy consumption and sufficient joint stiffness to ensure stable movement. Therefore, active joint stiffness should be evaluated and its relation with antagonistic muscle activations should be modeled in future work, similar to the explorations of $[16,23]$.

We note that for a given criterion with a set of weight coefficients, the optimization result was not unique, but instead could be affected by the initialization. Nevertheless, for the same initialization and the same convergence criteria, the optimization result was unique.

During the experimental validation on real subjects, the results obtained for subject 6-left leg with a time-invariant model (Fig. 6, left column) illustrated the overall satisfactoriness of the synthesized FES patterns, with an angular tracking error of $8.46 \%$. In addition, the leg movements respected the dynamics of the desired trajectory, which is enough for several tasks that do not need very accurate tracking.

As we can see in Fig. 6c, even a simulated trajectory cannot exactly track the reference trajectory, although the simulated one was obtained from the same model used in the optimization. This means that the reduced musculoskeletal system (with only one muscle) is limited in tracking the chosen reference trajectory, and the use of antagonistic muscle may be required. We also noted that the greatest errors appeared during the first stimulation pattern. This transitory phenomenon at the beginning of stimulation was observed for some subjects but did not affect the behavior thereafter. We believe that this phenomenon was transitory since, when the same stimulation pattern was applied a second time, the measured trajectory was close to the reference one, as expected. In $s u b$ ject 9-right leg, the time-varying behavior appeared to show a higher amplitude of system response than that simulated with the time-invariant model. Therefore, this behavior variation may be due to unexpected fatigue during the identification session or to a variation in electrode placement. Comparison results before and after the $F_{c m}$ update showed a better prediction of the knee joint angle with the updated model (Fig. 6, right column). Indeed, the NRMSD of errors, which was about $55.9 \%$ with the first model, became about $13.2 \%$ with the updated one (see Table 2). However, a bigger prediction error with the updated model appeared at the last motion pattern (Fig. 6f), probably due to fatigue during the synthesis validation session.

Table 2 summarizes the errors for all subjects with the first-identified model and the updated one, thanks to the $F_{\mathrm{cm}}$ parameter update. It indicates the improvement in the synthesis results, with the average of the NRMSD errors dropping from $31.42 \%$ with the first model to $13.96 \%$ with the updated model. We can also note that the updated model of subject 6-left leg did not improve the results (see Table 2), since the behavior was time-invariant.

These encouraging results are similar to those obtained in [9], where an open-loop control was applied to two subjects and the normalized RMS error of the knee angle was about $15 \%$. Given that the average range of movement was $40^{\circ}$ in our results, the RMS error is about $5.6^{\circ}$ which is also comparable to the results obtained in other works, such as [22], where the average error obtained from four subjects was about $4.1^{\circ}$, and [5], where the RMS errors obtained from two subjects were $5.14^{\circ}$ and $7.14^{\circ}$. However, in these last two works, the stimulation was manually adjusted in the first work [22] and controlled through a Neuro-PID controller in the second work [5], which highlights the originality of our FES synthesis method and results.

Table 2 shows that most of the subjects required a model update to improve the synthesis results. However, the low number of tests does not give us a precise idea about the update procedure needed in future tests.

The $F_{\mathrm{cm}}$ values presented in Table 2 show a decrease between the first-identified model and the updated one in subject 7-left leg, subject 9-left leg and subject 10-right 
leg. This indicates the appearance of fatigue during the synthesis session compared with the identification session. Conversely, the $F_{c m}$ value increased in subject 9-right leg because of fatigue during the identification session and the recovery of muscle capacities during the synthesis session.

The current approach of model updating does not solve the problem of time-varying behavior; however, it provides some explanations and suggests directions for exploring ways to overcome this problem and generalize FES synthesis. This variation required updating the synthesized FES patterns, since the current ones were optimized from the model identified before the synthesis validation session. Therefore, one of the considerable limitations of this work is related to the limitation of the open-loop control application. Indeed, this control strategy is affected by the accuracy of the identification procedure and by the presence of time-varying parameters, as it has been pointed out in previous works $[5,9,22]$.

Overall, the current experimental results show that our FES synthesis protocol is efficient when the system is time-invariant, although it presents limitations when the behavior is time-varying. In order to improve the results in the presence of these variations, we suggest several directions for exploration in future works. The first one is to find a simple relationship between the parameter variation and its effect on the synthesized FES patterns to maintain the same behavior. Thus, this variation should be used to adjust the FES patterns obtained with the initial model. The other option is online synthesis of the FES patterns, which requires online adjustment of the varying parameter. This requires reducing the time calculation of the identification and optimization procedure.

Our experimental validation, for example, focused on the knee joint controlled by the quadriceps muscle only. Thus, without an antagonistic muscle, there was no redundancy problem to solve. In this case, the synthesis presented a solution of numerical inversion of a highly nonlinear system. This was the first experimental validation of FES synthesis, which will be generalized thereafter. Therefore, the synthesis based on tracking of a reference trajectory poses no problem of muscular energy consumption, since there is no antagonistic muscle to increase the co-contraction level. This can be a solution for tracking a trajectory without excessively increasing the cocontraction level and therefore the energy consumption. However, we must acknowledge that in the absence of redundancy, the musculoskeletal system loses part of its natural capacities for joint stiffness control and stability, as discussed above. Therefore, a future research perspective is to include the antagonistic hamstring muscles in the validation procedure in order to experimentally validate the FES synthesis with a co-activated musculoskeletal system.

\section{Conclusion}

This work explored the synthesis of the optimal FES patterns required for restoration of a desired movement in subjects with spinal cord injury. It consisted of optimizing FES patterns that minimize a criterion and with respect to certain physiological and mechanical constraints. In the current work, we focused on the knee joint, which is controlled by two antagonistic stimulated muscles, the quadriceps and the hamstrings.

In the first step of the work, we tested the FES synthesis in simulation by exploring three strategies for movement restoration in the leg. Depending on the synthesis strategy, we minimized a criterion based on the muscular energetic consumption alone or combined with a tracking error between a reference movement and the predicted movement. The simulation results were successful in that they respected the defined tasks and constraints. The results were then compared in terms of the co-contraction level and energy consumption of torque and muscle activities. The comparisons revealed a high level of muscular energy consumption related to a high co-contraction rate when a trajectory is tracked. However, co-contraction increases joint stiffness, which may contribute to the stability of the movement against external perturbations like ground contact.

Therefore, a compromise between minimizing muscular consumption and ensuring joint stability during tasks should be explored in future works. With the first simulation method, we explored the effects of the optimization criterion weight coefficients. The results showed that a higher weight of muscular activities minimizes the co-contraction rate, and thus the muscular energy consumption, but reduces the accuracy of trajectory tracking. Here also, the choice of the weight coefficients requires a compromise between energy consumption and accuracy.

In the second step of this work, the FES synthesis was experimentally tested on four subjects with complete spinal cord injury. This experimental validation used a reduced subject-specific model, with parameters identified in our previous work on the knee joint controlled with quadriceps muscle only. The results of applying the synthesized FES patterns to the quadriceps showed good agreement between the desired and the measured leg movements. These successful results demonstrated the efficiency of our method of FES synthesis for movement rehabilitation when no time-variation appears in the musculoskeletal system behavior. However, the presence of time-varying behavior marked the limit of this off-line FES synthesis, which required a model update during the synthesis validation process. On the other hand, the simulated behavior with the updated model, based on a measured time-variation, showed the efficiency of the maximal isometric force $F_{\mathrm{cm}}$ update to take into account the time-varying behavior. It 
also provided some explanations and opened perspectives for exploring ways to overcome this problem and improve the efficiency of FES synthesis in these particular cases.

Therefore, two issues should be explored in future works: updating the synthesized FES patterns from the parameter variation when time-variation behavior is detected and online FES synthesis, which requires online updating of the $F_{\mathrm{cm}}$ parameter. This solution requires reducing the calculation time with respect to real-time constraints.

Future works should also include the antagonistic hamstring muscles in the experimental procedure. This would improve the accuracy of the tracking trajectory and increase the stability of the joint against external perturbations. However, a new experimental identification protocol adapted to the hamstring muscles would have to be included and a function for co-contraction level would need to be taken into account.

Acknowledgments The authors would like to thank the patients for their participation and patience, and also Maria Papaiordanidou, Robin Passama and Patrick Benoit for their help during the experiments.

\section{References}

1. Benoussaad M, Poignet P, Guiraud D (2007) Optimal patterns synthesis approach for knee motion under functional electrical stimulation. In: 12th Annual Conference of the International FES Society, Philadelphia, PA, USA

2. Benoussaad M, Poignet P, Guiraud D (2008) Optimal functional electrical stimulation patterns synthesis for knee joint control. In: Intelligent robots and systems, 2008. IROS 2008. IEEE/RSJ International Conference on Nice, pp 2386-2391. doi:10.1109/I ROS.2008.4651112

3. Benoussaad M, Poignet P, Hayashibe M, Azevedo-Coste C, Fattal C, Guiraud D (2013) Experimental parameter identification of a multi-scale musculoskeletal model controlled by electrical stimulation: application to patients with spinal cord injury. Med Biol Eng Comput 51:617-31. doi:10.1007/s11517-013-1032-y

4. Chae J, Kilgore K, Triolo R, Creasey G, DiMarco A (2004) Functional neuromuscular stimulation. In: DeLisa J, Gans D (eds) Rehabilitation medicine: principles and practice, 4th edn. J. B. Lippincott Company, Philadelphia, pp 1405-1425

5. Chang GC, Luh JJ, Liao GD, Lai JS, Cheng CK, Kuo BL, Kuo TS (1997) A Neuro-control system for the knee joint position control with quadriceps stimulation. IEEE Trans Rehabil Eng 5:2-11

6. Chizeck H, Kobetic R, Marsolais EB, Abbas JJ, Donner IH, Simon E (1988) Control of functional neuromuscular stimulation systems for standing and locomotion in paraplegics. Proc IEEE 76:1155-1165

7. Dariush B, Parnianpour M, Hemami H (1998) Stability and a control strategy of a multilink musculoskeletal model with applications in FES. IEEE Trans Biomed Eng 45:3-14

8. El Makssoud H, Guiraud D, Poignet P, Hayashibe M, Wieber PB, Yoshida K, Azevedo-Coste C (2011) Multiscale modeling of skeletal muscle properties and experimental validations in isometric conditions. Biol Cybern 105:121-138. doi:10.1007/ s00422-011-0445-7

9. Ferrarin M, Palazzo F, Riener R, Quintern J (2001) Model-based control of FES-induced single joint movements. IEEE Trans Rehabil Eng 9:245-257
10. Franken DHM, Veltink PPH, Tijsmans IR, Nijmeijer DH, Boom PHBK (1993) Identification of passive knee joint and shank dynamics in paraplegics using quadriceps stimulation. IEEE Trans Rehabil Eng 1:154-164

11. Guiraud D, Stieglitz T, Koch KP, Divoux JL, Rabischong P (2006) An implantable neuroprosthesis for standing and walking in paraplegia: 5-year patient follow-up. J Neural Eng 3:268-275

12. Hatze H (1978) A general myocybernetic control model of skeletal muscle. Biol Cybern 157:143-157

13. Hatze H (1981) Myocybernetic control models of skeletal muscle. University of South Africa, South Africa

14. Hawkins D, Hull M (1990) A method for determining lower extremity muscle-tendon lengths during flexion/extension movements. J Biomech 23:487-494

15. Hill AV (1938) The heat of shortening and the dynamic constants of muscle. R Soc Lond Proc Ser B 126:136-195

16. Hogan N (1984) Adaptive control of mechanical impedance by coactivation of antagonist muscles. IEEE Trans Automat Contr 29:681-690

17. Huxley AF (1957) Muscle structure and theories of contraction. Prog Biophys Biophys Chem 7:255-318

18. Khalil W, Dombre E (2004) Trajectory generation. In: Khalil W, Dombre E (eds) Modeling, identification and control of robots. Butterworth-Heinemann, Oxford, pp 313-345

19. Kobetic R, Marsolais B (1994) Synthesis of paraplegic gait with multichannel functional neuromuscular stimulation. IEEE Trans Rehabil Eng 2:66-79

20. Levy M, Mizrahi J, Susak Z (1990) Recruitment, force and fatigue characteristics of quadriceps muscles of paraplegics isometrically activated by surface functional electrical stimulation. $\mathrm{J}$ Biomed Eng 12:150-156. doi:10.1016/0141-5425(90)90136-B

21. Lin DC, Rymer WZ (1991) A quantitative analysis of pendular motion of the lower leg in spastic human subjects. IEEE Trans Biomed Eng 38:906-918. doi:10.1109/10.83611

22. McNeal D, Nakai R, Meadows P, Tu W (1989) Open-loop control of the freely-swinging paralyzed leg. IEEE Trans Biomed Eng 36:895-905

23. Migliore SA, Brown EA, DeWeerth SP (2005) Biologically inspired joint stiffness control. In: Proceedings of the 2005 IEEE international conference on robotics and automation, pp 4508-4513. doi:10.1109/ROBOT.2005.1570814

24. Popović D, Stein RB, Oguztöreli MN, Lebiedowska M, Jonić S (1999) Optimal control of walking with functional electrical stimulation: a computer simulation study. IEEE Trans Rehabil Eng 7:69-79

25. Riener R, Fuhr T (1998) Patient-driven control of FES-supported standing up: a simulation study. IEEE Trans Rehabil Eng 6:113-124

26. Riener R, Quintern J, Psaier E, Schmidt G (1996) Physiological based multi-input model of muscle activation. Neuroprosthetics 12:95-114

27. Stein RB, Zehr EP, Lebiedowska MK, Popović DB, Scheiner A, Chizeck HJ (1996) Estimating mechanical parameters of leg segments in individuals with and without physical disabilities. IEEE Trans Rehabil Eng 4:201-211

28. Veltink PH, Chizeck HJ, Crago PE, El-Bialy A (1992) Nonlinear joint angle control for artificially stimulated muscle. IEEE Trans Biomed Eng 39:368-380. doi:10.1109/10.126609

29. Winters JM (1990) Hill-based muscle models: a systems engineering perspective. In: Winters JM, Woo SY (eds) Chapter 5 Multiple muscle systems: biomechanics and movement organization, New York: Springer, pp 69-93

30. Zhou BH, Katz SR, Baratta RV, Solomonow M, D’Ambrosia RD (1997) Evaluation of antagonist coactivation strategies elicited from electrically stimulated muscles under load-moving conditions. IEEE Trans Biomed Eng 44:620-633 